\title{
Kategorisierung von Röntgenbildern mit aktiven Konturmodellen
}

\author{
Jörg Bredno, Sebastian Brandt, Jörg Dahmen*, Berthold Wein ${ }^{\dagger}$ \\ und Thomas Lehmann \\ Institut für Medizinische Informatik \\ ${ }^{*}$ Lehrstuhl für Informatik VI \\ ${ }^{\dagger}$ Klinik für Radiologische Diagnostik \\ Rheinisch-Westfälische Technische Hochschule (RWTH), 52057 Aachen \\ Email: jbredno@mi.rwth-aachen.de
}

\begin{abstract}
Zusammenfassung. Für das Image Retrieval in Medical Applications (IRMA) müssen digitalen Radiographien automatisch Körperregionen zugeordnet werden. Experimentell werden Methoden zum formbasierten Image Retrieval auf radiologische Bilder angewendet. Es wird untersucht, ob die Umrißlinie dargestellter Körperteile mit einem Ballon-Modell aufgefunden werden kann. Anschließend werden semilokale invariante Signaturen ermittelt und in ihren Klassifikationseigenschaften mit invarianten Momenten und Fourier-Koeffizienten verglichen. Eine dem visuellen Eindruck entsprechende Konturfindung mit dem Ballon-Modell gelingt auf 496 von 1616 Radiographien, die Nearest-Neighbour-Klassifikation auf Basis der extrahierten Formmerkmale in sechs Kategorien erreicht bisher nur Klassifikationsraten von $65 \%$.
\end{abstract}

Schlüsselwörter: Image Retrieval, Klassifikation, aktive Kontur, Signatur, Invarianz

\section{$1 \quad$ Einleitung}

Im Rahmen des IRMA-Projektes (Image Retrieval in Medical Applications) werden unter anderem Methoden untersucht, um Bilder aus einem konventionellen Archiv automatisch in Kategorien bezüglich Körperregion, Aufnahmemodalität und -orientierung einzuteilen [1]. Einfache Klassifikationsmethoden für sekundär digitale Radiographien auf Basis deskriptiver Bildstatistiken wie Histogrammen oder Cooccurrence-Matrizen alleine erwiesen sich als nicht erfolgreich. Für den Betrachter ist ein wesentliches Kriterium zur Kategorisierung von Körperteilen die Form von dargestellten Organen oder Skelettabschnitten. Es existieren bereits verschiedene erfolgreiche Systeme zum formbasierten Image Retrieval [2, 3], wobei nach anderen Quellen Formmerkmale nicht zu guten Retrieval-Ergebnissen führen [4]. Experimentelle Aussagen über das vorliegende Bildmaterial sind bisher nicht bekannt. Im folgenden werden verschiedene Merkmale zur Formbeschreibung extrahiert und zur Klassifikation angewendet, um die Möglichkeiten einer automatischen Kategorisierung auf Grund von Forminformationen für Radiographien bewerten zu können. 


\section{Methode}

\subsection{Detektion der Umrißlinien}

Zur Detektion einer beliebig geformten Kontur, über deren Form kein a-prioriWissen vorliegt, wird ein Ballon-Modell eingesetzt. Dessen charakteristische Eigenschaft ist eine durch Innen- oder Außendruck modellierte Suchrichtung. Der Druck führt zu einer Bewegung von Knotenpunkten des Modells orthogonal zur aktuellen Richtung der Kontur. Damit sind Ballon-Modelle im Gegensatz zu vielen anderen aktiven Konturmodellen nicht von der Wahl einer der Lösung ähnlichen Startkontur abhängig.

Unser Ballon-Modell wurde bereits erfolgreich eingesetzt, um unterschiedlichstes Bildmaterial zu quantifizieren [5]. Es wird am Bildrand initialisiert und zieht sich um dargestellte Objekte unter Einfluß äußeren Drucks zusammen. Wir verwenden eine Zuordnung von Grauwerten zu Bildpotentialen, um die Stärke externer Einflüsse zu bestimmen. Diese Zuordnung wird nach einer Voranalyse des Histogramms so getroffen, daß alle Grauwerte im Bereich der ungeschwächten Filmbelichtung kein Potential erhalten, und daß die auf Kanten ausgeübte Kraft von maximalen Grauwerten doppelt so stark ist wie der Außendruck. Dieser läßt die Kontur schrumpfen, wenn nur geringe Bildpotentiale wirken. Grauwerte im Bereich metallischer Komponenten im Bild erhalten ebenfalls kein Potential.

Nach der Erstarrung der aktiven Kontur wird überprüft, welcher Prozentsatz der Kontur entlang metallischer Komponenten im Bild verläuft. Dieser Anteil wird durch eine Betrachtung der Grauwerte in einem schmalen Streifen außerhalb der Kontur bestimmt. Wenn er zehn Prozent überschreitet, werden weitere Iterationen mit veränderten Parametern durchgeführt, um die Kontur von diesen Gradienten zu lösen. Anschließend wird die Erkennung mit den ursprünglichen Einflußgrößen weitergeführt.

\subsection{Merkmale zur Formbeschreibung}

Zur Beschreibung einer gefundenen Umrißlinie wird eine Variante der semilokalen Signaturen verwendet, die invariant gegenüber affinen Transformationen ist. Diese Signaturen werden bereits für das Image Retrieval eingesetzt, wenn Teile einer Kontur verdeckt sind [2]. Unsere Motivation zum Einsatz dieser Merkmale ist nicht die teilweise Verdeckung, sondern die möglicherweise fehlerbehaftete Erkennung biologischer Objekte in medizinischem Bildmaterial. Zur Berechnung der Signaturen wird zunächst ein lineares Resampling der detektierten Umrißlinie auf $N$ gleichmäßig verteilte Knoten durchgeführt. Dann werden für jeden Knoten $n$ unterschiedliche Signaturwerte zum Skalenparameter $d$ ermittelt. Es sei $v_{i}$ ein Knoten der zyklischen Kontur mit $v_{i+N}=v_{i}$. Ein Signaturwert $s(i, d)$ für den Knoten $v_{i}$ zum Parameter $d$ wird ermittelt zu: 


$$
s(i, d)=\left\{\begin{array}{ccc}
\max _{i=0}^{N-1} s(i, d) & \forall & A\left(\triangle_{v_{i}, v_{i+2 d}, v_{i-d}}\right)=0 \\
\min _{i=0}^{N-1} s(i, d) & \forall & A\left(\triangle_{v_{i}, v_{i+d}, v_{i-2 d}}\right)=0 \\
\ln \left(\frac{A\left(\triangle_{v_{i}, v_{i+d}, v_{i-2 d}}\right)}{A\left(\triangle_{v_{i}, v_{i+2 d}, v_{i-d}}\right)}\right) & \text { sonst }
\end{array}\right.
$$

Ein Signaturwert ist also der natürliche Logarithmus des Flächenverhältnisses zweier Dreiecke, die neben dem Punkt $v_{i}$ entweder durch die Punkte $v_{i+d}, v_{i-2 d}$ oder $v_{i+2 d}, v_{i-d}$ gebildet werden. Falls eines dieser Dreiecke aus kollinearen Punkten besteht, werden für den mathematisch nicht definierten Ausdruck der höchste bzw. niedrigste in der Kontur tatsächlich auftretende Signaturwert verwendet. Die Signaturwerte $s(i, d)$ werden zur Signatur $S=\{s(i, d) \mid i \in[0, \ldots, N-1], d \in$ $[1, \ldots, n]\}$ der Kontur vereinigt.

Ein Distanzmaß $D\left(S_{1}, S_{2}\right)$ zwischen zwei Signaturen ist die minimale Cityblock-Distanz, die sich bei allen möglichen zyklischen Vertauschungen zweier Signaturen untereinander ergibt:

$$
D\left(S_{1}, S_{2}\right)=\min _{k=1 \vee k=-1} \min _{i=0}^{N-1} \sum_{j=0}^{N-1} \sum_{d=1}^{n}\left|s_{1}(j, d)-s_{2}(i+k \cdot j \bmod N, d)\right|
$$

Der Vergleich wird gleichläufig und gegenläufig durchgeführt, um die affine Invarianz der Signaturwerte auch für die Signaturen selbst zu erhalten. Um die große Variabilität biologischer Formen besser widerspiegeln zu können, wurde zusätzlich ein elastisches Distanzmaß $\tilde{D}\left(S_{1}, S_{2}\right)$ eingeführt. Dieses teilt die Signatur $S_{1}$ in Blöcke fester Größe, die dann mit bester Übereinstimmung auf die Signatur $S_{2}$ gelegt werden. Dabei darf zwischen den einzelnen Blöcken eine Überlappung oder eine Lücke bis zu einer vorgegebenen Größe auftreten.

Neben den semilokalen invarianten Signaturen werden zu den ermittelten Umrißlinien sieben invariante Momente und die längennormierten ersten neun Fourier-Koeffizienten ermittelt. Für diese RST-invarianten Merkmale wird die Cityblock-Distanz verwendet [3].

\subsection{Qualitätsbestimmung der Formmerkmale}

Um die unterschiedlichen Formmerkmale auf ihre Fähigkeit hin zu untersuchen, Umrißlinien auf Röntgenaufnahmen charakteristisch und diskriminant zu beschreiben, wurden der klinischen Routine 1616 Röntgenaufnahmen entnommen, für die eine a-priori-Zuordnung in die Kategorien Extremitäten, Wirbelsäule, Abdomen, Thorax, Mammographie und Schädel vorliegt. Die ermittelten Merkmale wurden getrennt voneinander zu Klassifikationsexperimenten eingesetzt. Dabei wurde eine 1-Nearest-Neighbour-Klassifikation verwendet, die Güte der Merkmale wurde durch Klassifikation nach der Leaving-One-Out-Methode bestimmt. Die 1-Nearest-Neighbour-Klassifikation wurde gewählt, um die Qualität der Merkmale zu evaluieren. Die Untersuchungen wurden sowohl für den 
Tabelle 1. Klassifikationsergebnisse für einzelne Merkmale

\begin{tabular}{lrr} 
Merkmal & Alle Konturen & Korrekte Konturen \\
\hline Fourier-Koeffizienten & $43,2 \%$ & $58,5 \%$ \\
Invariante Momente & $48,9 \%$ & $65,1 \%$ \\
Signaturen & n. bestimmt & $59,8 \%$ \\
Elastische Signaturen & n. bestimmt & $55.6 \%$ \\
\hline
\end{tabular}

kompletten Datensatz als auch nur für diejenigen Bilder, für die eine dem visuellen Eindruck entsprechende Konturdetektion erreicht wurde, durchgeführt.

\section{$3 \quad$ Ergebnisse}

Die Detektion der Umrißlinien und die Extraktion der Formmerkmale wurden in der IRMA-Entwicklungsumgebung [6] realisiert, um die folgenden Experimente durchzuführen. Für die invarianten Signaturen wurde $N=100$ und $d \in[1, \ldots, 5]$ verwendet. Für das elastische Distanzmaß wurden 10 Blöcke der Breite 10 verwendet, die sich um bis zu drei Punkte überlappen oder voneinander trennen können.

\subsection{Detektion der Umrißlinie}

Der Algorithmus zur Konturdetektion liefert ein Detektionsergebnis für 1506 der 1616 Radiographien. In den übrigen Aufnahmen schrumpft die Kontur zu einem Punkt zusammen, ohne signifikante Kanten im Bild aufgefunden zu haben. Eine manuelle Inspektion der detektierten Konturen ergab nur für 496 Aufnahmen ein Segmentierungsergebnis, das den visuellen Eindruck eines Betrachters widerspiegelt. Die Erkennung ist zwar sehr robust gegen inhomogene Belichtungen und auch metallische Abdeckungen im Bild, aber die bisher gewählte Zuordnung von Grauwerten zu Bildpotentialen ist nur für Skelettradiographien geeignet. Aufnahmen mit hohem Weichgewebeanteil und metallischen Objekten im Bild, beispielsweise Mammographien mit aufgelegten Bleilettern, werden mit der gewählten Adaption falsch parametriert, so daß keine akzeptable Konturdetektion erfolgt.

\subsection{Klassifikationsergebnisse}

Die erfolgversprechenden ersten Ergebnisse mit geringerer Bildzahl ließen sich für die Gesamtmenge der vorliegenden Bilder nicht wiederholen. Ein Vergleich der Methoden für alle korrekten Konturen ergibt, daß die invarianten Momente das beste Klassifikationsergebnis $(65,1 \%)$ ergeben, mit Fourier-Koeffizienten werden $58,5 \%$ der Bilder korrekt in eine der sechs Kategorien eingeordnet. Die nicht gravierende Verbesserung gegenüber der Klassifikation aller 1506 detektierten Konturen (48,9\% bzw. 43,2\%) zeigt, daß diese Merkmale auch für Radiographien nicht mit der kognitiven Wahrnehmung korrelieren. 
Ein Vergleich der Signaturen mit elastischem Distanzmaß $\tilde{D}\left(S_{1}, S_{2}\right)(55,6 \%)$ bringt keine Verbesserung gegenüber dem statischen Maß $D\left(S_{1}, S_{2}\right)(59,8 \%)$ (Tab. 1). Insgesamt schneiden die affin invarianten Signaturen nicht besser ab als die RST-invarianten Merkmale. Nach unserer Interpretation führt insbesondere die Invarianz gegenüber der Stauchung und damit der Verlust der Information über die Proportion von Objekten zu vielen Fehlklassifikationen. Neben dem 1Nearest-Neighbour-Klassifikators wurden bisher keine weiteren Klassifikationsmethoden angewendet, das Hauptaugenmerk liegt auf einem fairen Vergleich der unterschiedlichen Merkmale.

\section{Diskussion}

Sowohl die Erkennung von Umrißlinien als auch deren Kategorisierung werden noch nicht mit befriedigender Qualität durchgeführt. Bisher ließ sich der methodische Ansatz formbasierter Image-Retrieval-Systeme [2,3] nicht erfolgreich auf medizinisches Bildmaterial übertragen. Die schwierige Erkennung ist insbesondere darauf zurückzuführen, daß die Einstellungen für die Belichtung eines Röntgenfilms nicht vorgenommen werden, um Kontrast und Schärfe der Umrisse dargestellter Objekte zu optimieren. Aufgabe ist die Darstellung bestmöglicher Informationen für eine Diagnose.

Die Kategorisierung im Rahmen des IRMA-Projektes wird mit der Kombination verschiedenster Merkmale erfolgen [1]. Die Forminformationen versprechen zwar nicht einzeln, nach der erfolgten Qualitätsbestimmung aber in Kombination mit anderen Merkmalen eine zufriedenstellende automatische Kategorisierung.

\section{Literatur}

1. Lehmann T, Wein B, Dahmen J, Bredno J, Vogelsang F, Kohnen M: Ein strukturiertes Konzept zum inhaltsbasierten Zugriff auf medizinische Bildarchive. (Dieser Band).

2. Kliot M, Rivlin E: Invariant-based shape retrieval in pictorial databases. Computer Vision and Image Understanding, 71(2), pp 182-197, 1998.

3. Huang DH, Huang CL: A content-based image retrieval system. Image and Vision Computing, 16(3), pp 149-163, 1998.

4. Dahmen J, Lehmann T, Spitzer K, Ney H: Image Retrieval für klinische Bildatenbanken. In: Lehmann T et al. (Hrsg.) Bildverarbeitung für die Medizin 1998, pp 442-446, Springer-Verlag, Berlin, 1998.

5. Metzler V, Bredno J, Lehmann T, Spitzer K: A deformable membrane for the segmentation of cytological samples. Proc. SPIE 3338, pp 1246-1257, 1998.

6. Bredno J, Vogelsang V, Dahmen J et al.: Eine Entwicklungsumgebung für die interdisziplinäre Zusammenarbeit bei der Entwicklung des Image-RetrievalSystems IRMA. In: Evers H et al. (Hrsg.) Bildverarbeitung für die Medizin 1999, pp 362-366, Springer-Verlag, Berlin, 1999. 